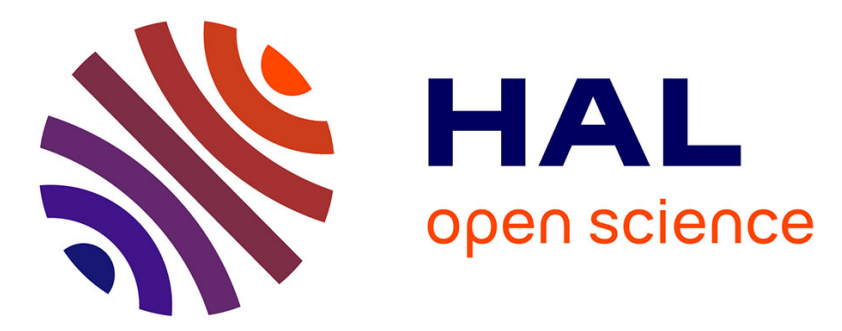

\title{
On the Cramer Rao bound and maximum likelihood in passive time delay estimation for complex signals
}

\author{
Jean-Pierre Delmas, Yann Meurisse
}

\section{To cite this version:}

Jean-Pierre Delmas, Yann Meurisse. On the Cramer Rao bound and maximum likelihood in passive time delay estimation for complex signals. ICASSP '12: IEEE International Conference on Acoustics, Speech and Signal Processing, Mar 2012, Kyoto, Japan. pp.3541-3544, 10.1109/ICASSP.2012.6288681 . hal-00746455

\section{HAL Id: hal-00746455 \\ https://hal.science/hal-00746455}

Submitted on 16 Dec 2020

HAL is a multi-disciplinary open access archive for the deposit and dissemination of scientific research documents, whether they are published or not. The documents may come from teaching and research institutions in France or abroad, or from public or private research centers.
L'archive ouverte pluridisciplinaire HAL, est destinée au dépôt et à la diffusion de documents scientifiques de niveau recherche, publiés ou non, émanant des établissements d'enseignement et de recherche français ou étrangers, des laboratoires publics ou privés. 


\title{
ON THE CRAMER RAO BOUND AND MAXIMUM LIKELIHOOD IN PASSIVE TIME DELAY ESTIMATION FOR COMPLEX SIGNALS
}

\author{
Jean Pierre Delmas and Yann Meurisse \\ TELECOM SudParis, Département CITI, UMR CNRS 5157 \\ 91011 Evry, France
}

\begin{abstract}
This paper is devoted to time delay estimation for wide sense stationary complex circular or noncircular Gaussian signals. Using a theorem by Whittle that we have extended to complex data, closed-form expressions of the Cramer Rao bound (CRB) are given for the time delay alone in presence of nuisance parameters. In particular, we prove that the CRB for the time delay is weakly reduced for noncircular signals w.r.t. circular signals, except for very low signal to noise ratios (SNR), for which the $\mathrm{CRB}$ for rectilinear signals is half of the $\mathrm{CRB}$ for circular signals. Then, the maximum likelihood (ML) estimate that extends the generalized cross correlation (GCC) estimate is derived.
\end{abstract}

Index Terms - Time delay estimation, circular and noncircular complex signals, Whittle formula, cross correlation.

\section{INTRODUCTION}

Time delay estimation plays an important role in radar, sonar, and seismology for localizing radiating sources. It has been extensively studied in the past decade (e.g., [1], [2] and the references therein), but to the best of our knowledge, only for real-valued signals.

Here we focus on the time delay estimation based on the continuous-time complex envelopes issued from two separate sensors in passive systems. To make the model mathematically tractable, these signals are assumed wide sense stationary complex circular or noncircular Gaussian. The main aim of this paper is to give closed-form expressions of the CRB for the time delay alone in presence of nuisance parameters and to derive the ML estimator for time delay given signal and noise spectra.

The paper is organized as follows. Section 2 introduces the data model and assumptions. Section 3 extends to complex data a theorem by Whittle [3] for the derivation of the Fisher Information matrix (FIM). In Section 4, closed-form expressions of the CRB are given for the time delay alone in presence of nuisance parameters for circular and noncircular signals. In Section 5, the ML estimate for time delay given signal and noise spectra that extends the generalized cross correlation (GCC) introduced in [2] is derived. Finally
Section 6 illustrates the comparison of the CRB for the time delay for noncircular signals w.r.t. circular signals for both white spectra and white complementary spectra.

\section{DATA MODEL AND ASSUMPTIONS}

A continuous-time signal $r(t)$ whose spectrum is bandlimited around $f_{0}$ emanating from a remote source and monitored in the presence of noise at two separated sensors can be modeled as

$$
r_{1}(t)=r(t)+w_{1}(t) \text { and } r_{2}(t)=a r\left(t-\tau_{0}\right)+w_{2}(t),
$$

where $a$ and $\tau_{0}$ denote the relative attenuation and delay of $r(t)$ w.r.t. the first sensor. After bandpass filtering and down conversion of these two signals to baseband, the in-phase and quadrature components are paired to obtain the complex envelopes:

$$
\begin{aligned}
& x_{1}(t)=s\left(t-\tau_{g}\left(f_{0}\right)\right)+n_{1}(t) \\
& x_{2}(t)=\kappa s\left(t-\tau_{g}\left(f_{0}\right)-\tau_{0}\right)+n_{2}(t)
\end{aligned}
$$

where $r(t)=\Re\left(s(t) e^{i 2 \pi f_{0} t}\right), \kappa=a e^{-i 2 \pi f_{0}\left(\tau_{0}+\tau_{\phi}\left(f_{0}\right)\right)}$ and where $\tau_{\phi}\left(f_{0}\right)$ and $\tau_{g}\left(f_{0}\right)$ are, respectively, the phase and group delays of the perfectly linear and identical passband filters at the output of the two sensors.

To make the derivation of the CRB on the delay $\tau_{0}$ mathematically tractable, the complex baseband signals $s(t), n_{1}(t)$ and $n_{2}(t)$ are assumed mutually uncorrelated, zero-mean Gaussian and wide-sense stationary (WSS) (i.e., their real and imaginary parts are jointly WSS). The noise components $n_{1}(t)$ and $n_{2}(t)$ are assumed circular, in contrast to $s(t)$ that is not necessarily circular ${ }^{1}$.

In this case, the statistical properties of the noise are characterized by the power spectral densities (PSD) $N_{1}(f)$ and $N_{2}(f)$ : Fourier transforms of $R_{n_{i}}(\tau)=\mathrm{E}\left(n_{i}(t) n_{i}^{*}(t-\tau)\right)$, $i=1,2$, whereas $s(t)$ needs both the PSD $S(f)$ and complementary PSD $C(f)$ : Fourier transforms of $R_{s}(\tau)=$ $\mathrm{E}\left(s(t) s^{*}(t-\tau)\right)$ and $C_{s}(\tau)=\mathrm{E}(s(t) s(t-\tau))$, respectively [4, Sec: 8.1$]$.

\footnotetext{
${ }^{1}$ Note that if $r(t)$ were assumed WSS, $s(t)$ would be WSS and circular, but if $s(t)$ is WSS, $r(t)$ is WSS or wide-sense cyclostationary with the cyclic frequency $2 f_{0}$, depending on whether $s(t)$ is circular or noncircular.
} 
We assume that $x_{1}(t)$ and $x_{2}(t)$ are bandlimited in $\left[-\frac{1}{2 T_{s}},+\frac{1}{2 T_{s}}\right]$ and sampled at the Shannon rate $1 / T_{s}$ giving the discrete-time processes $x_{i}(n)=x_{i}(t)_{\mid t=n T_{s}}, i=1,2$. For simplicity $T_{s}=1$ in the following. With these assumptions, the PSD $\mathbf{S}_{x}(f)$ and complementary PSD $\mathbf{C}_{x}(f)$ matrices of the 2-variate process $\mathbf{x}(n)=\left[x_{1}(n), x_{2}(n)\right]^{T}$ are given from (2) by

$$
\mathbf{S}_{x}(f)=\left[\begin{array}{cc}
S(f)+N_{1}(f) & \kappa^{*} S(f) e^{i 2 \pi f \tau_{0}} \\
\kappa S(f) e^{-i 2 \pi f \tau_{0}} & |\kappa|^{2} S(f)+N_{2}(f)
\end{array}\right]
$$

and

$$
\mathbf{C}_{x}(f)=\left[\begin{array}{cc}
C(f) & \kappa C(f) e^{i 2 \pi f \tau_{0}} \\
\kappa C(f) e^{-i 2 \pi f \tau_{0}} & \kappa^{2} C(f)
\end{array}\right]
$$

\section{COMPLEX WHITTLE FORMULA}

To derive the FIM for our complex data model (2), we have to extend the following theorem due to Whittle [3, th. 9] to complex-valued data.

Theorem Let $\mathbf{x}(n)$ be a real-valued discrete time WSS zeromean Gaussian multivariate process with spectrum $\mathbf{S}_{x}(f)$ that depends on the real-valued parameter $\boldsymbol{\theta}$. Then the elements $\mathbf{J}_{k, l}$ of the FIM associated with $N$ sample values of $\mathbf{x}(n)$ are asymptotically (w.r.t. to $N$ ), given by

$\mathbf{J}_{k, l}=\frac{N}{2} \int_{-1 / 2}^{+1 / 2} \operatorname{Tr}\left(\frac{\partial \mathbf{S}_{x}(f)}{\partial \theta_{k}} \mathbf{S}_{x}^{-1}(f) \frac{\partial \mathbf{S}_{x}(f)}{\partial \theta_{l}} \mathbf{S}_{x}^{-1}(f)\right) d f$.

Consider now a complex-valued WSS zero-mean Gaussian multivariate process $\mathbf{x}(n)$ with spectrum $\mathbf{S}_{x}(f)$ and complementary spectrum $\mathbf{C}_{x}(f)$. The Whittle formula (5) applies to $\overline{\mathbf{x}}(n) \stackrel{\text { def }}{=}\left[\begin{array}{l}\Re(\mathbf{x}(n)) \\ \Im(\mathbf{x}(n))\end{array}\right]=\frac{1}{2} \mathbf{U} \tilde{\mathbf{x}}(n)$ with $\mathbf{U} \stackrel{\text { def }}{=}\left[\begin{array}{cc}\mathbf{I} & \mathbf{I} \\ -i \mathbf{I} & i \mathbf{I}\end{array}\right]$ and $\tilde{\mathbf{x}}(n) \stackrel{\text { def }}{=}\left[\begin{array}{c}\mathbf{x}(n) \\ \mathbf{x}^{*}(n)\end{array}\right]$, whose spectra $\mathbf{S}_{\bar{x}}(f)$ and $\mathbf{S}_{\tilde{x}}(f)$ are related by $\mathbf{S}_{\bar{x}}(f)=\frac{1}{4} \mathbf{U} \mathbf{S}_{\tilde{x}}(f) \mathbf{U}^{H}$. Introducing the spectrum $\mathbf{S}_{\bar{x}}(f)$ in (5) and using $\operatorname{Tr}(\mathbf{A B})=\operatorname{Tr}(\mathbf{B A})$ with $\mathbf{U}^{-1}=$ $\frac{1}{2} \mathbf{U}^{H}$ prove that (5) still applies ${ }^{2}$ by replacing $\mathbf{S}_{x}(f)$ with $\mathbf{S}_{\tilde{x}}(f)$, where

$$
\mathbf{S}_{\tilde{x}}(f)=\left[\begin{array}{cc}
\mathbf{S}_{x}(f) & \mathbf{C}_{x}(f) \\
\mathbf{C}_{x}^{*}(-f) & \mathbf{S}_{x}^{*}(-f)
\end{array}\right] .
$$

In the particular case of circular complex data, the complementary spectrum $\mathbf{C}_{x}(f)$ is zero and it is straightforward to

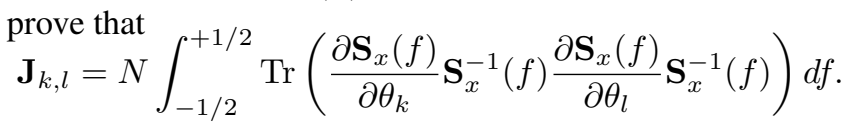

\section{CRAMER RAO BOUND}

Generally the spectrum $S(f)$ and the complementary spectrum $C(f)$ of $s(t)$ and the spectra $N_{1}(f)$ and $N_{2}(f)$ of the

\footnotetext{
${ }^{2}$ Note that the theorem also applies to our continuous-time process sampled at the Shannon rate.
}

noise are not perfectly known. $S(f)$ and $C(f)$ [resp., $N_{1}(f)$ and $N_{2}(f)$ ] depend on unknown parameters $\boldsymbol{\alpha}$ [resp., $\boldsymbol{\beta}$ ], which, along with the complex attenuation $\kappa$, comprise the nuisance parameters. Thus the unknown parameter of $\mathbf{S}_{\tilde{x}}(f)$ is $\boldsymbol{\theta}=\left(\tau_{0}, \Re(\kappa), \Im(\kappa), \boldsymbol{\alpha}^{T}, \boldsymbol{\beta}^{T}\right)^{T}$. In the sequel, we distinguish the circular case, where closed-form expressions are available and the noncircular case, where due to the inversion of the $4 \times 4$ matrix $\mathbf{S}_{\tilde{x}}(f)$, only numerical properties have been derived except in specific conditions.

\subsection{Circular case}

On differentiating $\mathbf{S}_{x}(f)$ and performing the required matrix inversion and multiplications of (5), we have proved in particular that

$$
\begin{aligned}
\mathbf{J}_{\tau_{0}, \tau_{0}} & =8 \pi^{2}|\kappa|^{2} N \int_{-1 / 2}^{+1 / 2} \frac{f^{2} S^{2}(f)}{\Delta_{\kappa}(f)} d f \\
\mathbf{J}_{\tau_{0}, \Re(\kappa)} & =-4 \pi \Im(\kappa) N \int_{-1 / 2}^{+1 / 2} \frac{f S^{2}(f)}{\Delta_{\kappa}(f)} d f \\
\mathbf{J}_{\tau_{0}, \Im(\kappa)} & =-4 \pi \Re(\kappa) N \int_{-1 / 2}^{+1 / 2} \frac{f S^{2}(f)}{\Delta_{\kappa}(f)} d f \\
\mathbf{J}_{\tau_{0}, \alpha} & =\mathbf{J}_{\tau_{0}, \beta}=0,
\end{aligned}
$$

but $\mathbf{J}_{\Re(\kappa), \alpha}, \mathbf{J}_{\Im(\kappa), \alpha}, \mathbf{J}_{\Re(\kappa), \beta}$ and $\mathbf{J}_{\Im(\kappa), \beta}$ are nonzero, with $\Delta_{\kappa}(f) \stackrel{\text { def }}{=} \operatorname{det}\left(\mathbf{S}_{x}(f)\right)=\left(S(f)+N_{1}(f)\right)\left(S(f)+N_{2}(f)\right)-$ $|\kappa|^{2} S^{2}(f)$. After deriving the other terms of $\mathbf{J}$, (which are not given here for want of space), we note that $\mathbf{J}$ does not depend on $\tau_{0}$. Consequently $\operatorname{CRB}\left(\tau_{0}\right)=\left(\mathbf{J}^{-1}\right)_{1,1}$ does not depend on $\tau_{0}$ either.

The FIM of the joint estimation of $\left(\tau_{0}, \kappa\right)$ and the parameters $(\alpha, \beta)$ of spectra $S(f), N_{1}(f)$ and $N_{2}(f)$ is not block diagonal. Thus, the CRB for $\tau_{0}$ is smaller or equal in the case of known spectra, despite (10). Generally $\mathbf{J}_{\tau_{0}, \Re(\kappa)} \neq 0$ and $\mathbf{J}_{\tau_{0}, \Im(\kappa)} \neq 0$. So, the CRB for $\tau_{0}$ is smaller or equal when $\kappa$ is known. However, we note from (8) and (9), that in the case where the spectra $S(f), N_{1}(f)$ and $N_{2}(f)$ are all even ${ }^{3}$, $\mathbf{J}_{\tau_{0}, \Re(\kappa)}=\mathbf{J}_{\tau_{0}, \Im(\kappa)}=0$ and thus

$$
\operatorname{CRB}\left(\tau_{0}\right)=\mathbf{J}_{\tau_{0}, \tau_{0}}^{-1}=\left(8 \pi^{2}|\kappa|^{2} N \int_{-1 / 2}^{+1 / 2} \frac{f^{2} S^{2}(f)}{\Delta_{\kappa}(f)} d f\right)^{-1},
$$

which is naturally a decreasing function of the attenuation $a=|\kappa|$ that vanishes for non overlapping signal $S(f)$ and noise $\left(N_{1}(f), N_{2}(f)\right)$ spectra. For $a=|\kappa|=1$, (11) is half of the CRB for $\tau_{0}$ obtained in [5, (10)] for the delay estimation associated with the real-valued model (1) without any nuisance parameter (i.e., with $a=1$ ).

Finally, we note that for model (2) with no attenuation $(a=1)$, using now the unknown parameter $\boldsymbol{\theta}=$

\footnotetext{
${ }^{3}$ This is the practical case where $r(t)$ is a modulation with carrier $f_{0}$ and $w_{1}(t)$ and $w_{2}(t)$ are white noises in the bandwidth of $r(t)$.
} 
$\left(\tau_{0}, \operatorname{Arg}(\kappa), \boldsymbol{\alpha}^{T}, \boldsymbol{\beta}^{T}\right)^{T}$, we have proved that the CRB for $\tau_{0}$ remains unchanged w.r.t. the previous parametrization for arbitrary spectra.

\subsection{Noncircular case}

To derive interpretable closed-form expressions of the CRB for $\tau_{0}$ in the noncircular case, we have to consider the following specific four conditions:

(i) The complementary spectrum $C(f)$ of $s(t)$ is related to its spectrum $S(f)$ by the relation $C(f)=\rho S(f) e^{2 i \phi}$ with $\rho \in[0,1]$ and where $\rho$ and $\phi$ can be interpreted as, respectively, the second-order noncircularity modulus and phase of $s(t)$ [6]. For example, for $s(t)$ circular, $\rho=0$ and for $s(t)$ rectilinear (i.e., $\left.s(t)=|s(t)| e^{i \phi}\right), \rho=1$.

(ii) The spectra of $n_{1}(t)$ and $n_{2}(t)$ are identical: $N_{1}(f)=$ $N_{2}(f)=N(f)$.

(iii) The known spectra $S(f)$ and $N(f)$ are even.

(iv) There is no attenuation: $a=|\kappa|=1$.

In these conditions, $\boldsymbol{\theta}=\left(\tau_{0}, \operatorname{Arg}(\kappa), \rho, \phi\right)^{T}$ and we have proved from (5), where $\mathbf{S}_{x}(f)$ is replaced by $\mathbf{S}_{\tilde{x}}(f)$ given by (6)

$$
\begin{gathered}
\mathbf{J}_{\tau_{0}, \tau_{0}}=8 \pi^{2} N \\
\int_{-1 / 2}^{+1 / 2} \frac{f^{2} S^{2}(f)\left(2 S(f)\left(1-\rho^{2}\right)+N(f)\left(1+\rho^{2}\right)\right)}{N(f)(2 S(f)(1+\rho)+N(f))(2 S(f)(1-\rho)+N(f))} d f \\
\mathbf{J}_{\tau_{0}, \operatorname{Arg}(\kappa)}=\mathbf{J}_{\tau_{0}, \rho}=\mathbf{J}_{\tau_{0}, \phi}=0 .
\end{gathered}
$$

Consequently $\operatorname{CRB}\left(\tau_{0}\right)=\mathbf{J}_{\tau_{0}, \tau_{0}}^{-1}$, that gives respectively for $\rho=0$ (consistent with (11)) and $\rho=1$

$$
\begin{aligned}
& \operatorname{CRB}\left(\tau_{0}\right)=\left(8 \pi^{2} N \int_{-1 / 2}^{+1 / 2} \frac{f^{2} S^{2}(f)}{N(f)(2 S(f)+N(f))} d f\right)^{-1} \\
& \operatorname{CRB}\left(\tau_{0}\right)=\left(16 \pi^{2} N \int_{-1 / 2}^{+1 / 2} \frac{f^{2} S^{2}(f)}{N(f)(4 S(f)+N(f))} d f\right)^{-1} .
\end{aligned}
$$

Furthermore, we have numerically noted (by extensive scenarios) that if the condition (iv) is withdrawn, all the nuisance parameters (including now $|\kappa|$ ) remain decoupled in the FIM.

\section{ML ESTIMATOR OF THE TIME DELAY}

To derive the ML estimator of the time delay based on the continuous-time observation $\left(x_{1}(t), x_{2}(t)\right)_{t \in[-T / 2, T / 2]}$, we use its equivalence with the sequence of the Fourier coefficients of $x_{j}(t), j=1,2$

$$
X_{j}(k)=\frac{1}{T} \int_{-T / 2}^{+T / 2} x_{j}(t) e^{-i 2 \pi k t / T} d t .
$$

Note that the sequence $\left(X_{1}(k), X_{2}(k)\right)_{k \in \mathbb{Z}}$, as linear transform of $\left(x_{1}(t), x_{2}(t)\right)$, is complex zero-mean Gaussian distributed as $\left(x_{1}(t), x_{2}(t)\right)_{t \in[-T / 2, T / 2]}$ and circular if $s(t)$ is circular. For real-valued $x_{j}(t)$, a well known theorem of statistics [7, Sec. 6.4] asserts that these Fourier coefficients associated with different frequencies $k / T$ are asymptotically (w.r.t. to $T$ ) uncorrelated. More precisely, if $T$ is large compared to the delay $\tau_{0}$ plus the correlation time of signal and noise we have

$$
\mathrm{E}\left(X_{i}(k) X_{j}^{*}(l)\right) \approx \frac{\delta_{k, l}}{T} S_{x_{i}, x_{j}}\left(\frac{k}{T}\right),
$$

$i, j=1,2$, where $\delta_{k, l}$ is the Kronecker delta function and $S_{x_{i}, x_{j}}(f)$ are the components of the spectra $\mathbf{S}_{x}(f)(3)$.

For complex-valued $x_{j}(t)$, we have proved that (13) remains valid and

$$
\mathrm{E}\left(X_{i}(k) X_{j}(l)\right) \approx \frac{\delta_{k,-l}}{T} C_{x_{j}, x_{i}}\left(\frac{k}{T}\right),
$$

where $C_{x_{i}, x_{j}}(f)$ are the components of the complementary spectra $\mathbf{C}_{x}(f)(4)$. We note that in contrast to the circular case, the Fourier coefficients are no longer independent in the noncircular case.

As $\mathbf{x}(t)$ is bandlimited to $\left[-\frac{1}{2 T_{s}},+\frac{1}{2 T_{s}}\right]$, the sequence $\left(X_{1}(k), X_{2}(k)\right)$ can be truncated to $k \in\{-K, \ldots,+K\}$ (with $K=\left\lfloor T / 2 T_{s}\right\rfloor$ ) and the probability density function of the complex Gaussian distributed vector $\mathbf{x}=\left[X_{1}(-K), X_{2}(-K)\right.$, .., $\left.X_{1}(K), X_{2}(K)\right]^{T}$ given the unknown parameter $\boldsymbol{\theta}=$ $(\tau, \kappa)$ (we suppose in this section that $S(f), C(f), N_{1}(f)$ and $N_{2}(f)$ are perfectly known) is written as

$$
p(\mathbf{x} ; \boldsymbol{\theta})=\frac{1}{\pi^{2(2 K+1)} \operatorname{det}^{1 / 2}\left(\frac{1}{T} \mathbf{S}_{\bar{x}}\right)} e^{-\frac{T}{2} \widetilde{\mathbf{x}}^{H} \mathbf{S}_{\bar{x}}^{-1} \widetilde{\mathbf{x}}},
$$

where $\widetilde{\mathbf{x}} \stackrel{\text { def }}{=}\left[\mathbf{x}^{T}, \mathbf{x}^{H}\right]^{T}$ and $\mathbf{S}_{\bar{x}}$ is the $2(2 K+1) \times 2(2 K+1)$ matrix

$$
\mathbf{S}_{\bar{x}}=\left[\begin{array}{cc}
\operatorname{diag}\left(\mathbf{S}_{x}\left(\frac{k}{T}\right)\right) & \operatorname{antidiag}\left(\mathbf{C}_{x}\left(-\frac{k}{T}\right)\right) \\
\operatorname{antidiag}\left(\mathbf{C}_{x}^{*}\left(\frac{k}{T}\right)\right) & \operatorname{diag}\left(\mathbf{S}_{x}^{*}\left(-\frac{k}{T}\right)\right)
\end{array}\right]
$$

Because we have proved that $\operatorname{det}\left(\mathbf{S}_{\bar{x}}\right)$ does not depend on $\tau$ but only on $\kappa$, the ML estimate $\widehat{\tau}_{0}$ is given by $\widehat{\tau}_{0}=$ $\arg \max _{\tau}\left[L_{\widehat{\kappa}}(\tau)\right]$ where $L_{\widehat{\kappa}}(\tau)=-T \widetilde{\mathbf{x}}^{H} \mathbf{S}_{\bar{x}}^{-1} \widetilde{\mathbf{x}}, \widehat{\kappa}$ is either the ML estimate of $\kappa$, or the known parameter $\kappa$ and $\mathbf{S}_{\tilde{x}}(f)$ depends on $\tau$ and $\widehat{\kappa}$. Noting that $\mathbf{S}_{\bar{x}}^{-1}$ is structured as $\mathbf{S}_{\bar{x}}$, i.e., $\mathbf{S}_{\bar{x}}^{-1}=\left[\begin{array}{cc}\operatorname{diag}\left(\mathbf{A}_{x}\left(\frac{k}{T}\right)\right) & \operatorname{antidiag}\left(\mathbf{B}_{x}\left(-\frac{k}{T}\right)\right) \\ \operatorname{antidiag}\left(\mathbf{B}_{x}^{*}\left(\frac{k}{T}\right)\right) & \operatorname{diag}\left(\mathbf{A}_{x}^{*}\left(-\frac{k}{T}\right)\right)\end{array}\right]$ where $\mathbf{A}_{x}(f)$ and $\left.\mathbf{B}_{x}(f)\right)$ are rational fractions of $\left(\mathbf{S}_{x}(f), \mathbf{C}_{x}(f)\right)$ (not given here for want of space), $L_{\widehat{\kappa}}(\tau)$ can be approximated for $T \gg T_{s}$ by

$$
L_{\widehat{\kappa}}(\tau) \approx-\int_{-1 / 2 T_{s}}^{1 / 2 T_{s}} \widetilde{\mathbf{x}}^{H}(f)\left[\begin{array}{ll}
\mathbf{A}_{x}(f) & \mathbf{B}_{x}(-f) \\
\mathbf{B}_{x}^{*}(f) & \mathbf{A}_{x}^{*}(-f)
\end{array}\right] \widetilde{\mathbf{x}}(f) d f
$$

where $\widetilde{\mathbf{x}}(f)=\left[\mathbf{x}^{T}(f), \mathbf{x}^{H}(-f)\right]^{T}$ with $\mathbf{x}(f)=\int_{-T / 2}^{+T / 2} \mathbf{x}(t) e^{-i 2 \pi f t} d t$. Using the periodogram estimates $\widehat{S}_{x_{2}, x_{1}}(f)=\frac{1}{T} x_{2}(f) x_{1}^{*}(f)$ and $\widehat{C}_{x_{2}, x_{1}}(f)=\frac{1}{T} x_{2}(f) x_{1}(f)$ 
of respectively the cross spectrum and complementary spectrum of $x_{2}(t)$ and $x_{1}(t)$ (with $\left.\mathbf{x}(f)=\left[x_{1}(f), x_{2}(f)\right]^{T}\right),(15)$ can be written in the following form

$$
\begin{aligned}
L_{\widehat{\kappa}}(\tau) \approx \int_{-1 / 2 T_{s}}^{1 / 2 T_{s}} \Re\left(a_{\widehat{\kappa}}(f) \widehat{S}_{x_{2}, x_{1}}(f) e^{i 2 \pi f \tau}\right. \\
\left.+b_{\widehat{\kappa}}(f) \widehat{C}_{x_{2}, x_{1}}(f) e^{i 2 \pi f \tau}\right) d f(16)
\end{aligned}
$$

where $a_{\widehat{\kappa}}(f)$ and $b_{\widehat{\kappa}}(f)$ are rational functions in $S(f), C(f)$, $N_{1}(f), N_{2}(f)$ and $\widehat{\kappa}$ (which are not given here for want of space).

The ML estimate $\widehat{\tau}_{0}$ given by the maximization of (16) extends the GCC estimate introduced by Knapp et al. [2] in the real case. It consists in widely filtering $x_{1}(t)$ and $x_{2}(t)$, cross correlating these outputs and then maximizing the real part of this cross correlation. We note that for white spectra and complementary spectra specified in Section 6), $\widehat{\tau}_{0}$ is given by

$$
\widehat{\tau}_{0}=\arg \max _{\tau} \Re\left(a_{1}(\widehat{\kappa}) \widehat{R}_{x_{2}, x_{1}}(\tau)+b_{1}(\widehat{\kappa}) \widehat{C}_{x_{2}, x_{1}}(\tau)\right),
$$

with $\widehat{R}_{x_{2}, x_{1}}(\tau)=\int_{-T / 2}^{T / 2} x_{2}(t) x_{1}^{*}(t-\tau) d t, \widehat{C}_{x_{2}, x_{1}}(\tau)=$ $\int_{-T / 2}^{T / 2} x_{2}(t) x_{1}(t-\tau) d t$, where $x_{1}(t)$ and $x_{1}(t)$ are zero outside $[-T / 2, T / 2] . a_{1}(\widehat{\kappa})$ and $b_{1}(\widehat{\kappa})$ are functions of $\widehat{\kappa}$ that are not given here for want of space. For $s(t)$ circular the maximization of (16) and (17) reduce, respectively, to

$$
\begin{gathered}
\widehat{\tau}_{0}=\arg \max _{\tau} \int_{-1 / 2 T_{s}}^{1 / 2 T_{s}} \frac{S(f)}{\Delta_{\kappa}(f)} \Re\left(\widehat{\kappa}^{*} \widehat{S}_{x_{2}, x_{1}}(f) e^{i 2 \pi f \tau}\right) d f \\
\widehat{\tau}_{0}=\arg \max _{\tau} \int_{-1 / 2 T_{s}}^{1 / 2 T_{s}} \Re\left(\widehat{\kappa}^{*} \widehat{S}_{x_{2}, x_{1}}(f) e^{i 2 \pi f \tau}\right) d f, \quad(18)
\end{gathered}
$$

in contrast to the real case, both require the knowledge of $\widehat{\kappa}$. In particular we note that from (18), despite $\left|R_{x_{2}, x_{1}}(\tau)\right|=$ $a\left|R_{s}\left(\tau-\tau_{0}\right)\right| \leq a R_{s}(0)$, the ML estimate $\widehat{\tau}_{0}$ is not given by $\arg \max _{\tau}\left|\widehat{R}_{x_{2}, x_{1}}(\tau)\right|$.

\section{NUMERICAL ILLUSTRATIONS}

To gain some insight into relation (12), we consider here the special case of subsection 4.2 in which the signal and noise are ideal white processes, i.e.,

$$
\begin{gathered}
S(f)=\left\{\begin{array}{ll}
\sigma_{s}^{2}, & |f| \leq 1 / 2 \\
0, & |f|>1 / 2
\end{array}, \quad C(f)= \begin{cases}\rho e^{2 i \phi} \sigma_{s}^{2}, & |f| \leq 1 / 2 \\
0, & |f|>1 / 2\end{cases} \right. \\
N(f)=\left\{\begin{array}{ll}
\sigma_{n}^{2}, & |f| \leq 1 / 2 \\
0, & |f|>1 / 2
\end{array} .\right.
\end{gathered}
$$

In that case, the nuisance parameters of the spectra are $\alpha=$ $\left(\sigma_{s}^{2}, \rho, \phi\right)^{T}$ and $\beta=\sigma_{n}^{2}$ and we have proved that in addition to (12) $\mathbf{J}_{\tau_{0}, \sigma_{s}^{2}}=\mathbf{J}_{\tau_{0}, \sigma_{n}^{2}}=0$ and the CRB for $\tau_{0}$ that depends only on $\rho$ and the SNR $r=\sigma_{s}^{2} / \sigma_{n}^{2}$, is monotonically decreasing with both $\rho$ and $r$.

$$
\operatorname{CRB}^{\rho}\left(\tau_{0}\right)=\frac{3}{2 \pi^{2} N r^{2}} \frac{(2 r(1+\rho)+1)(2 r(1-\rho)+1)}{2 r\left(1-\rho^{2}\right)+\left(1+\rho^{2}\right)},
$$

for which

$$
\operatorname{CRB}^{0}\left(\tau_{0}\right)=\frac{3}{2 \pi^{2} N r}\left(2+\frac{1}{r}\right)
$$

and

$$
\operatorname{CRB}^{1}\left(\tau_{0}\right)=\frac{3}{4 \pi^{2} N r}\left(4+\frac{1}{r}\right)
$$

for $\rho=0$ and $\rho=1$, respectively.

Fig. 1 plots the ratio $\operatorname{CRB}^{\rho}\left(\tau_{0}\right) / \operatorname{CRB}^{0}\left(\tau_{0}\right)$ w.r.t. to $\rho$ for different values of the SNR and for $a=1$ (deduced from (19)). We note that for arbitrary $a$, the behavior of this ratio (deduced from the numerical evaluation of $\mathbf{J}_{\tau_{0}, \tau_{0}}^{-1}$ ) w.r.t. $\rho$ is very similar with now $r$ defined by $\left(1+a^{2}\right) \sigma_{s}^{2} / \sigma_{n}^{2}$.

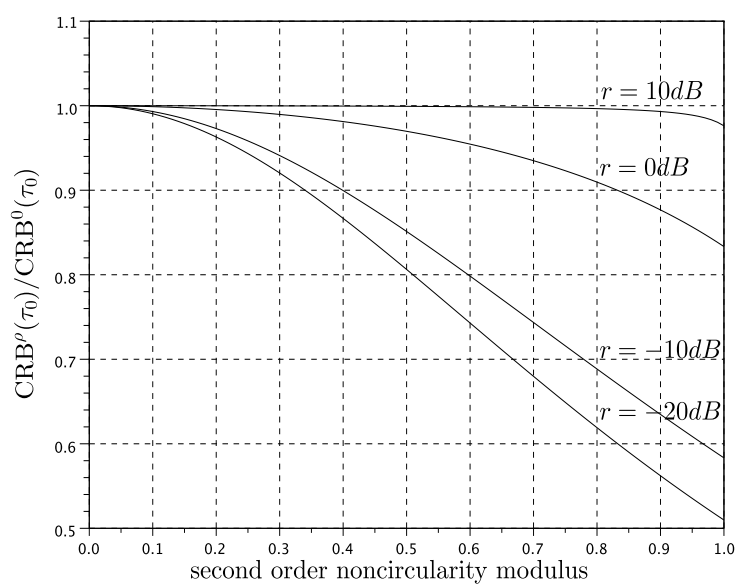

Fig.1 $\operatorname{CRB}^{\rho}\left(\tau_{0}\right) / \mathrm{CRB}^{0}\left(\tau_{0}\right)$ w.r.t. $\rho$ for different values of the SNR.

We see that the CRB for $\tau_{0}$ is weakly reduced for noncircular signals $s(t)$ w.r.t. to circular signals, except for very low SNR, for which the CRB for rectilinear signals is half of the CRB for circular signals, consistent with (20) and (21).

\section{REFERENCES}

[1] W.R. Hahn and S.A. Tretter, "Optimum processing for delayvector estimation in passive signal arrays", IEEE Trans. Inform. Theory, vol. 19, no. 5, pp. 608-614, Sept. 1973.

[2] C.H. Knapp and G.C. Carter, "The generalized correlation method for estimation of time delay", IEEE Trans. ASSP, vol. 24, no. 4, pp. 320-327, August 1976.

[3] P. Whittle, "The analysis of multiple stationary time series", $J$. Royal statist. Soc., vol. 15, pp. 125-139, 1953.

[4] P.J. Schreier and L.L. Scharf, Statistical signal processing of complex-valued data: the theory of improper and noncircular signals, Cambridge University Press, 2010.

[5] B. Friedlander, "On the Cramer Rao bound for time delay and Doppler estimation”, IEEE Trans. Information Theory, vol. 30, no. 3, pp. 575-580, May 1984.

[6] H. Abeida and J.P. Delmas, "MUSIC-like estimation of direction of arrival for non-circular sources," IEEE Trans. Signal Process., vol. 54, no. 7, pp. 2678-2690, July 2006.

[7] W.B. Davenport and W.L. Root, An Introduction to the Theory of Random Signals and Noise, New York: McGraw-Hill, 1958. 